\title{
A Method for Gait Events Detection based on Low Spatial Resolution Pressure Insoles data
}

\author{
F. Salis ${ }^{1,2}$, S. Bertuletti ${ }^{1,2}$, T. Bonci' ${ }^{3}$ U. Della Croce $^{1,2}$, C. Mazzà ${ }^{3}$, A. Cereatti ${ }^{2,4}$ \\ ${ }^{1}$ Department of Biomedical Sciences, University of Sassari, Sassari, Italy; ${ }^{2}$ Interuniversity Centre of Bioengineering \\ of the Human Neuromusculoskeletal System, Sassari, Italy; ${ }^{3}$ Insigneo Institute and Department of Mechanical \\ Engineering, University of Sheffield, Sheffield, UK; ${ }^{4}$ Department of Electronics and Telecommunications, \\ Politecnico di Torino, Torino, Italy.
}

\begin{abstract}
The accurate identification of initial and final foot contacts is a crucial prerequisite for obtaining a reliable estimation of spatio-temporal parameters of gait. Well-accepted gold standard techniques in this field are force platforms and instrumented walkways, which provide a direct measure of the foot-ground reaction forces. Nonetheless, these tools are expensive, non-portable and restrict the analysis to laboratory settings. Instrumented insoles with a reduced number of pressure sensing elements might overcome these limitations, but a suitable method for gait events identification has not been adopted yet. The aim of this paper was to present and validate a method aiming at filling such void, as applied to a system including two insoles with 16 pressure sensing elements (element area $=310 \mathrm{~mm}^{2}$ ), sampling at $100 \mathrm{~Hz}$. Gait events were identified exploiting the sensor redundancy and a cluster-based strategy. The method was tested in the laboratory against force platforms on nine healthy subjects for a total of 801 initial and final contacts. Initial and final contacts were detected with low average errors of (about $20 \mathrm{~ms}$ and $10 \mathrm{~ms}$, respectively). Similarly, the errors in estimating stance duration and step duration averaged $20 \mathrm{~ms}$ and less than $10 \mathrm{~ms}$, respectively. By selecting appropriate thresholds, the method may be easily applied to other pressure insoles featuring similar requirements.
\end{abstract}

E-mail addresses: fsalis1@uniss.it (F. Salis), sbertuletti@uniss.it (S. Bertuletti), t.bonci@sheffield.ac.uk (T. Bonci), dellacro@uniss.it (U.D.Croce), c.mazza@sheffield.ac.uk (C. Mazzà), andrea.cereatti@polito.it (A. Cereatti).

\section{Keywords:}

Gait analysis, wearable sensors, pressure insoles, locomotion, gait events

Published in: Journal of Biomechanics 127(2021) 110687

https://doi.org/10.1016/j.jbiomech.2021.110687 


\subsection{INTRODUCTION}

The gait cycle represents the functional element of walking, traditionally identified by the initial contact (IC) of the foot with the ground and the following IC of the same foot (Della Croce et al., 2018; Whittle, 1993). A direct approach to detect these gait events (GEs) is by using force platforms (FPs) and instrumented walkways. These provide a direct measure of forces resulting from the foot-ground interaction, thus representing a gold standard for GEs detection. However, both devices are non-portable, expensive and require an appropriate laboratory environment, therefore constraining the analysis to few strides and/or straight walks (Adkin et al., 2000). Moreover, laboratory analysis only allows for the assessment of walking capacity, which should ideally be complemented with continuous daily living measures of mobility performance to obtain a thorough assessment (World Health Organization, 2007; Rochester et al., 2020). In this perspective, wearable inertial measurement units (IMUs) are the key to enable gait analysis in real-world scenarios as GEs can be identified from the accelerations and angular velocities signals recorded by two units attached to the ankles/feet (Mariani et al., 2012; Trojaniello et al., 2014). However, being the latter an indirect method, processing algorithms performance may be affected by errors, and it should, therefore, be regarded as a silver standard solution.

Foot switches are an effective alternative to estimate GEs and their use has been explored in several studies over the last decades (Agostini et al., 2013; Bae et al., 2011; Hausdorff et al., 1995; Kong et al., 2009; Skelly et al., 2001). The foot switch technology, however, generally includes only two or three sensing elements, which require a proper positioning under the foot. Due its low spatial sensor resolution, the approach does not allow to identify the specific area of the sole-ground contact and, in turn, it may also affect the GEs temporal resolution. This is even more true in case of pathological gait (i.e., pronation, supination, equine gait, foot drop, shuffling children with cerebral palsy), for which few sensors are not sufficient (Smith et al., 2016). Another attractive option is represented by plantar pressure insoles, based on different technologies and sensors configurations (e.g., Tekscan $^{\circ}$ F-Scan ${ }^{\circ}$ System; Novel $^{\circ}$ Pedar ${ }^{\circ}$ System, etc.). However, these devices are specifically conceived for highresolution pressure mapping applications and generally include a dense grid of sensors (from 99 to 960 sensing elements) which inevitably lead to higher costs and complexity in terms of data management and reading, but which are not strictly necessary for simple GEs estimations.

In this study we propose an original method for GEs detection, based on the use of instrumented insoles, each including only sixteen force-sensing resistor elements (pressure insoles, PIs). The implemented algorithm exploits the number of sensors by using a cluster-based approach to describe foot-ground contacts in a finer way and avoid missed and extra GEs, providing information about foot positioning. The method was tested against FPs in the laboratory using data collected on healthy subjects.

\subsection{METHODS}

\subsubsection{System Description and GEs algorithm}

Two plantar PIs (mod. YETI, 221e S.r.I., Padua, Italy; 16 sensing elements; element area $=310 \mathrm{~mm}^{2} ; \mathrm{fs}=100$ $\mathrm{Hz}$; ground reaction force threshold $=5 \mathrm{~N}$ ) were used in this study, with a design similar to that adopted by Ciniglio et al. 2021. Each sensing element is constituted by a force sensing resistor, exhibiting a resistance value inversely proportional to the applied force. The output is expressed as voltage (full-scale voltage value $V_{F S}=2.8 \mathrm{~V}$ ). Each pressure insole is connected to a central processing unit, which also includes a magneto-IMU (Figure 1) that is not used for this study. Data is recorded by an ultra-low-power microcontroller and stored in an on-board flash storage. 


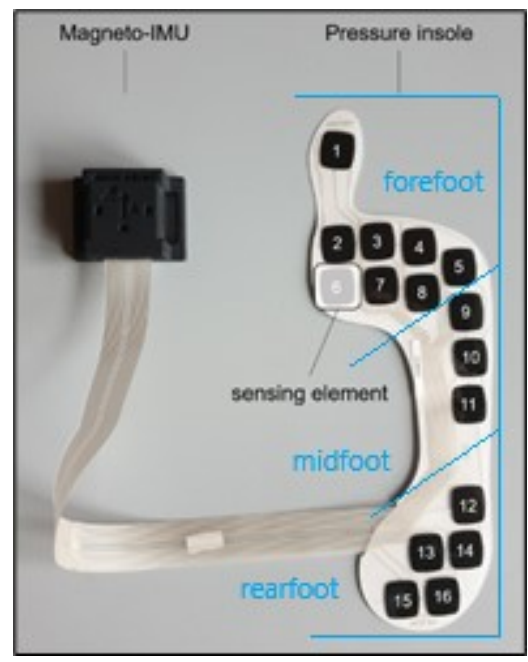

Figure 1: Magneto-IMU and pressure insole used for the right foot.

The PI signals processing algorithm is described by the following steps (Figure 2):

(i) Pre-processing.

$\mathrm{PI}$ signals are normalised with respect to $\mathrm{V}_{\mathrm{Fs}}$, expressed in normalised units (nu), and then filtered using a 5-points non-linear median filter to have a smoothing effect while enhancing edges (Stork et al., 2003);

(ii) Detection and selection of instants of rising and falling edges.

For each of the filtered PI signals $\mathrm{X}_{\mathrm{i}}(\mathrm{t})$, where $\mathrm{i}=1, . ., 16$ represents the $\mathrm{i}$-th $\mathrm{PI}$ signal, a first derivative approach (Hopkins, 2001) is applied to detect rising and falling edges. Edges are identified from $\dot{X}_{i}(t)$ using a peak detection approach (Benocci et al., 2009) with an amplitude threshold defined as $T_{1}=5 n$, being $n$ the signal noise amplitude as computed in static conditions (in this study, we used $\mathrm{Th}_{1}=0.05 \mathrm{nu}$ ). For each PI signal, rising edges are identified as positive peaks $>\mathrm{Th}_{1}$ and the corresponding time instants are organized in a vector $t_{R E, \text {. }}$. Similarly, falling edges are identified as negative peaks $<-\mathrm{Th}_{1}$ and the corresponding time instants are organized in a vector $t_{F E, i} \cdot$ Rising and falling edges are automatically checked, in terms of time distance and amplitude of the PI signal, to discard false positives. Figure 2 a shows an example of detection of a rising edge and a falling edge;

\section{(iii) Detection and selection of local minima (instants of rising and falling minima).}

The identification of the instants of rising and falling minima is performed by applying to $X_{i}(t)$ a threshold $T_{2}=$ $0.02 \mathrm{nu}$, using rising and falling edges as reference points (Hausdorff et al., 1995). In particular, each rising minima is identified as the first point with $X_{i}(t)<T h_{2}$ preceding the considered rising edge instant, while each falling minima is identified as the first point with $X_{i}(t)<T h_{2}$ after the considered falling edge instant. Rising minima instants and falling minima instants were organised in vectors, $t_{R M, i}$ and $t_{F M, i}$ respectively. Figure $2 a$ shows an example of detection of one rising minimum and one falling minimum;

(iv) Identification of activation/deactivation clusters.

Once the rising and falling minima instants are detected for all the PI signals, they are organised in chronological order in a unique vector ( $t_{R M}$ and $t_{F M}$ respectively), also noting the corresponding sensing element number in another vector $\left(s_{R M}\right.$ and $\left.s_{F M}\right)$. This step is needed to group the instants of rising/falling minima corresponding to the same foot contact, i.e. the PI sensing elements which activate/deactivate together when the foot hits the ground. An activation cluster is identified imposing that the time distance between consecutive instants of $t_{R M}$ is lower than $\mathrm{Th}_{3}=0.4 \mathrm{~s}$. Then, a deactivation cluster includes the instants of $t_{F M}$ between two consecutive activation clusters. For each cluster, the minima instants and the sensing elements numbers are saved (A_cluster /D_cluster $r_{\mathrm{j}}$, where $\mathrm{j}=\mathrm{j}$-th activation/deactivation cluster).

Figure $2 \mathrm{~b}$ shows an example of one activation cluster and one deactivation cluster. 
(v) Identification of IC/FC (final contact) intervals and definition of IC/FC events.

A foot-ground contact interval is defined when at least three sensing elements of the PI belonging to the same spatial neighbourhood are consecutively activated and deactivated, i.e. correspond to three consecutive minima belonging to the same cluster (A_cluster for ICs and D_cluster for FCs). For each PI's sensing element, the neighbourhood consists of those sensing elements which are spatially close to the considered unit (Figure 1) (e.g. for the sensing element no. 12, the neighbourhood includes sensing elements 11,13,14,15, 16; further details are reported in Appendix B). In fact, it is reasonable to assume that, when an IC or FC occurs, the sensing elements which refer to the same anatomically functional area of foot sole are activated or deactivated, respectively. Each IC interval is identified starting from the first rising minima of an activation cluster; while each FC interval is identified starting from the last falling minima of a deactivation cluster.

Finally, each IC is assumed to coincide with the rising minimum instant corresponding to the third sequentially activated sensing elements within the considered IC interval. Likewise, each FC is assumed to coincide with the falling minimum instant corresponding to the third sequentially deactivated sensing elements within the considered FC interval. Figure $2 \mathrm{c}$ shows an example of one IC interval and one FC interval.

A workflow of the algorithm can be found in Appendix A.
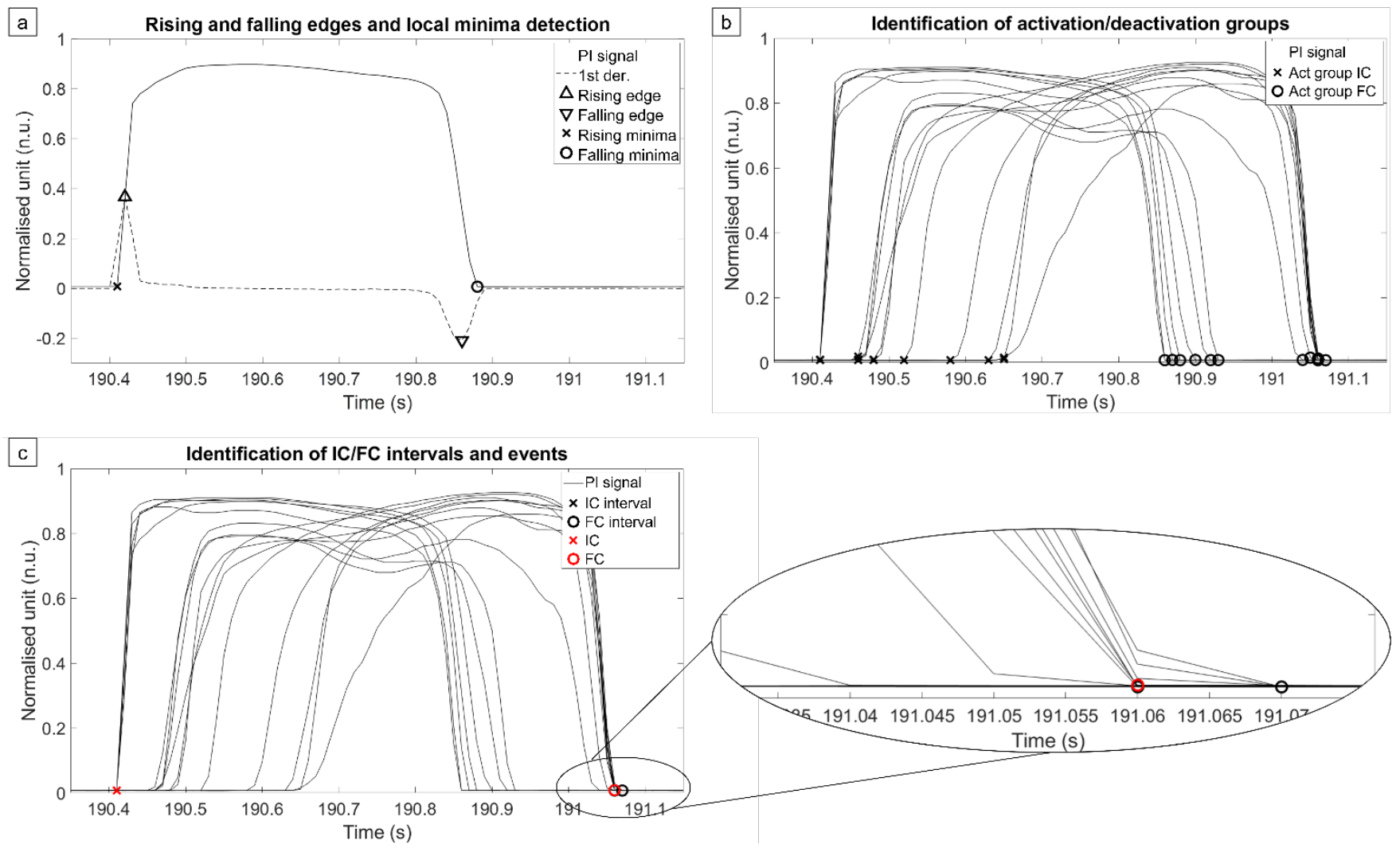

Figure 2: Principal steps of the algorithm shown for one stance. a) Detection and selection of rising and falling edges and local minima (rising and falling minima) for each PI signal; b) Identification of one activation/deactivation cluster on PI signals; $\mathrm{c}$ ) Identification of IC/FC intervals and definition of IC and FC events on PI signals.

\subsubsection{Experimental setup}

The validation experiments involved nine healthy participants ( 5 females and 4 males; age $25.4 \pm 1.3$ years, shoe size $40.5 \pm 4.1 \mathrm{EU}$ ) and took place at the University of Sassari (Italy). All participants signed an informed consent approved by the IRB before taking part to the study. PIs were inserted in participants' shoes and central processing units were clipped over the instep (Figure 3). The only specific requirement for the shoes was to avoid 
knee-high boots. Data from two FPs (AMTI, Massachusetts, USA; fs $=1000 \mathrm{~Hz}$ ) were acquired through a motion capture system also including video recordings (Vicon Vue, $\mathrm{fs}=50 \mathrm{~Hz}$ ). Data from PIs and FPs were synchronized using an additional central processing unit as external trigger, connected to the motion capture system via cable. Each participant was asked to walk for six minutes back and forth at comfortable speed, stepping on the FPs as many times as possible.
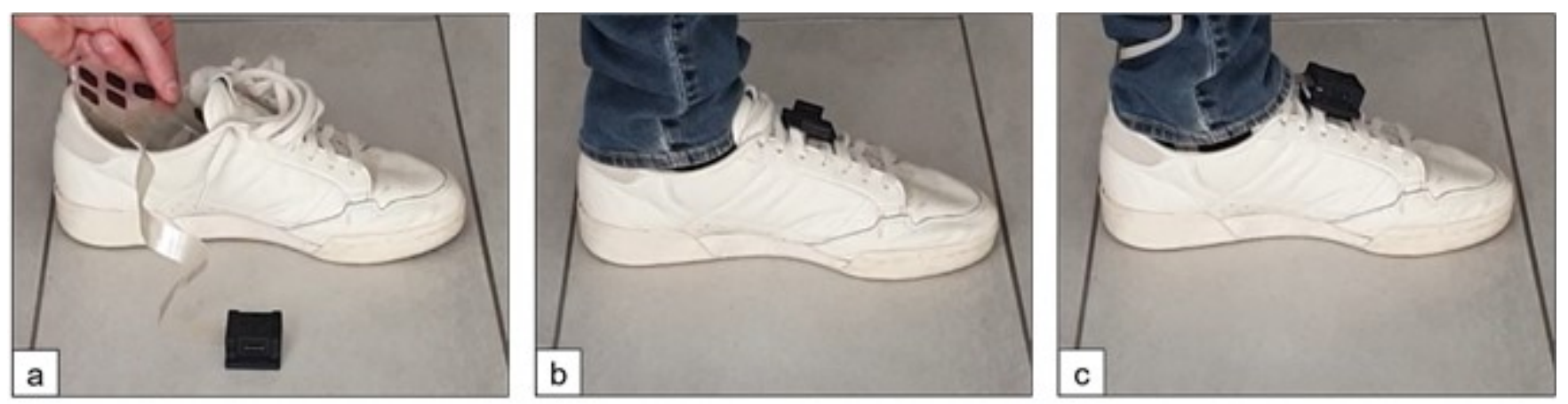

Figure 3: a) PI positioning inside the shoe; b) Clip attached to shoe laces; c) Final sensors positioning with magneto-IMU fixed to the clip.

\subsubsection{Data processing}

For each subject, a preliminary visual inspection of the "good strides" (entire foot on the FP during stance phase) was performed using video recordings. Then, FP data were down-sampled to $100 \mathrm{~Hz}$. A pre-processing procedure was applied for the synchronisation of PIs measurements (started via BLE protocol, v. 4.1) with the FP data, using the time vector provided by the trigger to interpolate the data.

The GEs detection algorithm results were compared with those obtained from the FPs (ground reaction force threshold $=25 \mathrm{~N}$ ) in terms of average root mean square (RMS) error, bias and standard deviation (SD) error computed over the stances of all participants. An example of IC and FC detection from both $\mathrm{PI}$ and FP is shown in Figure 4.
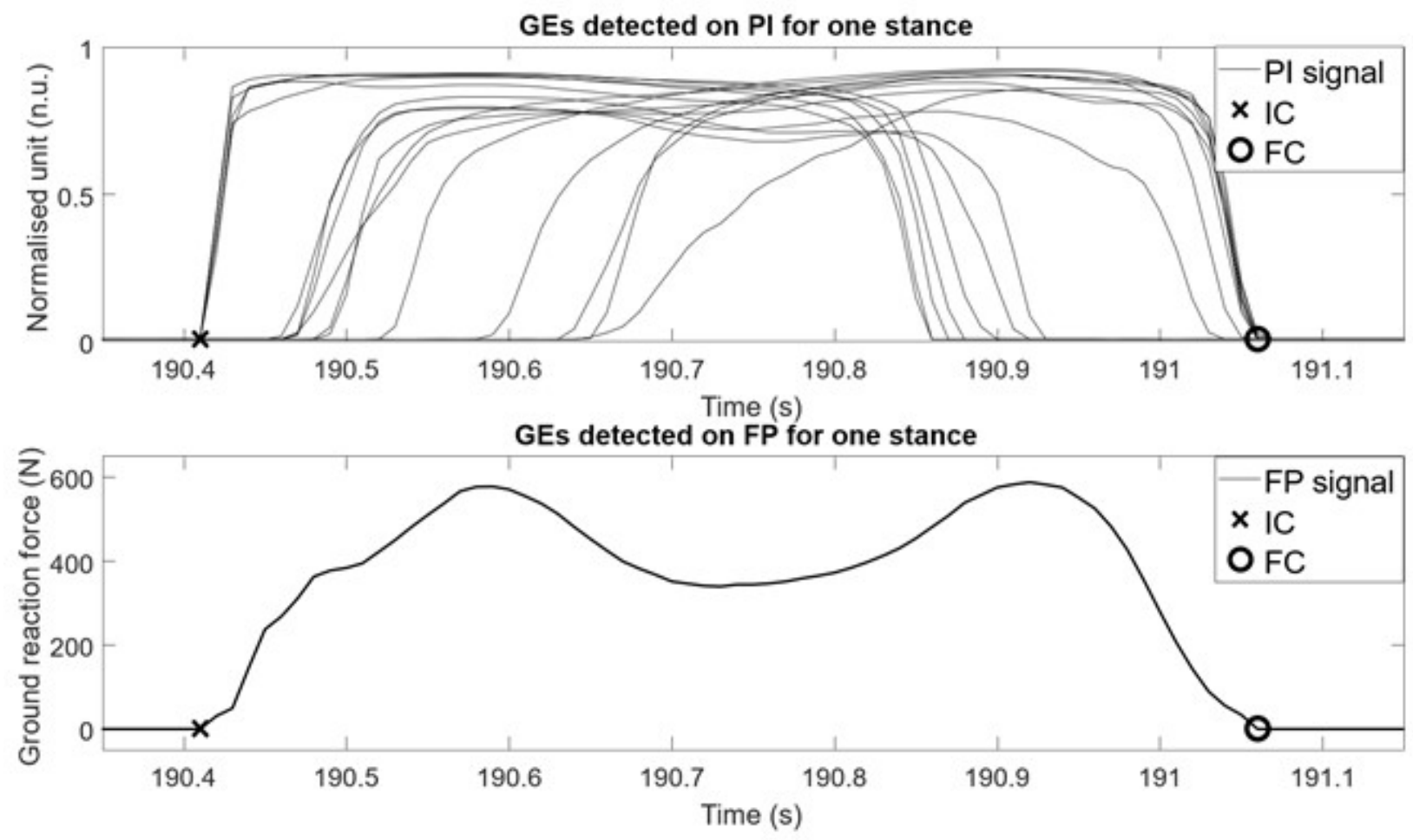

Figure 4: Gait events (GEs) detection from both pressure insole (PI) and force plate (FP). 


\subsection{RESULTS}

RMS error, bias and SD error obtained from the comparison are reported in Table 1. A total of 801 ICs and 801 FCs were analysed (89 ICs and FCs on average for each participant), while errors on step duration were computed considering 315 steps in total. Average errors were lower than 10 ms for FCs, 20 ms for ICs, 20 ms for stance duration, less than $1 \mathrm{~ms}$ for step duration.

Table 1: RMS error, bias, and SD error

\begin{tabular}{|c|c|c|c|}
\hline Variable & Average RMS Error (ms; frames) & Average Bias (ms; frames) & Average SD Error (ms; frames) \\
\hline IC & $22 ; 2$ & $-21 ;-2$ & $7 ;<1$ \\
\hline FC & $18 ;<2$ & $3 ;<1$ & $12 ; 1$ \\
\hline Stance duration & $18 ;<2$ & $23 ; 2$ & $7 ;<1$ \\
\hline Step duration & $10 ; 1$ & $0 ;<1$ & $10 ; 1$ \\
\hline
\end{tabular}

\subsection{DISCUSSION}

GEs and temporal parameters obtained from the Pls showed a $100 \%$ correspondence with those estimated from the FPs. Low average RMS errors were obtained for stance duration ( $<20 \mathrm{~ms})$ and for both IC and FC events, ( $22 \mathrm{~ms}$ and $17 \mathrm{~ms}$, respectively). IC events, as detected by the proposed method were, on average, anticipated with respect to those detected by the FP (average bias $=21 \mathrm{~ms}$ ), while FC events were marginally delayed. A bias of $23 \mathrm{~ms}$ was obtained for stance duration. Very low values were obtained for the average SD error (7 ms for ICs, $12 \mathrm{~ms}$ for FCs and $7 \mathrm{~ms}$ for stance duration). For step duration, both RMS error and SD error were around one sample, while the average bias was zero.

Similar but slightly larger errors were reported by Catalfamo and colleagues (2008) using a F-Scan Mobile Tekscan pressure insole ( $22 \pm 9$ ms for ICs and $10 \pm 4$ ms for FCs). However, it should be noted that the proposed algorithm was successful in obtaining lower errors using a pressure insole with a much smaller number of sensing elements (16 vs 960) and using a lower sample-frequency $(100 \mathrm{~Hz}$ vs $200 \mathrm{~Hz}$ ), with clear advantages in terms of cost and efficiency.

In general, the majority of the methodological studies analysing the performance of different pressure insoles, focused on gait parameters other than ICs and FCs and reported larger errors (Agarwal et al., 2020; Braun et al., 2015; Carbonaro et al., 2016; Crea et al., 2014). For instance, the average error reported in Carbonaro et al. (2016) by comparing a commercial smart shoe including two force sensors (FootMov) against a motion capture system was $39 \pm 65 \mathrm{~ms}$ for stance duration. Often, a direct comparison with the results in the literature was not possible due to the lack of a gold standard (Benocci et al., 2009), adoption of manual labelling of the GE detection (Roth et al., 2018) or different research objectives (i.e., PI signals used only for activity recognition).

The low errors found for both ICS and FCs demonstrated that the combined use of low-cost PI and specific algorithms for signal processing are a good compromise between more complex solutions, such as high-resolution pressure mapping technology, and foot-switch systems with a low number of sensors. A notable feature of the proposed method is that it can be applied to other PIs having a sufficient number of sensing elements. The minimum sensor number and area would clearly depend on the shoe size of the subjects to analyse (e.g. children), however, we found that an activated/deactivated area of about $900 \mathrm{~mm}^{2}$ (area of three sensing unit of the PI) guaranteed for good results for both male and female adults. Having a sufficiently high number of sensors allows to describe the foot-ground contact in a comprehensive way and virtually recognise all the possible strategies of foot-floor contact. Last but not least, the PIs here used can be easily combined with IMUs as part of a multi-sensor wearable system, which could provide accurate temporal estimates and a for a more extensive gait assessment also in a free-living context. Further studies will focus on overcoming the limitations of having tested the proposed method only on healthy subjects and on straight walking. 


\section{DECLARATION OF COMPETING INTEREST}

The authors declare that there are no financial nor personal relationships that can lead to conflicts of interest.

\section{ACKNOWLEDGEMENT}

The work was supported by the MOBILISE-D (EU H2020, EFPIA, and IMI 2 Joint Undertaking; Grant no. 820820), the UK EPSRC (K03877X/1 and S032940/1) and the NIHR Sheffield BRC. The study sponsors were not involved in the study phases, in the writing of the manuscript and in the decision about its submission.

\section{REFERENCES}

el Achkar, C. M., Lenoble-Hoskovec, C., Paraschiv-lonescu, A., Major, K., Büla, C., Aminian, K., 2016. Instrumented shoes for activity classification in the elderly. Gait \& posture, 44, 12-17.

Adkin, A. L., Frank, J. S., Carpenter, M. G., Peysar, G. W., 2000. Postural control is scaled to level of postural threat. Gait \& Posture, 12.2, 87-93.

Agarwal, R., Aggarwal, A., \& Gupta, R., 2020. A Wireless Sensorized Insole Design for Spatio-Temporal Gait Analysis. Neurophysiology, 52.3, 212-221.

Agostini, V., Balestra, G., Knaflitz, M., 2013. Segmentation and classification of gait cycles. In Proceedings of IEEE Transactions on Neural Systems and Rehabilitation Engineering, 22.5, 946-952.

Bae, J., Tomizuka, M., 2011. Gait phase analysis based on a Hidden Markov Model. Mechatronics, 21.6, 961-970.

Benocci, M., Rocchi, L., Farella, E., Chiari, L., Benini, L., 2009. A Wireless System for Gait and Posture Analysis Based on Pressure Insoles and Inertial Measurement Units. In Proceedings of the 3rd International Conference on Pervasive Computing Technologies for Healthcare, 1-6.

Braun, B. J., Veith, N. T., Hell, R., Döbele, S., Roland, M., Rollmann, M., ... Pohlemann, T., 2015. Validation and reliability testing of a new, fully integrated gait analysis insole. Journal of foot and ankle research, 8.1, 1-7.

Carbonaro, N., Lorussi, F., \& Tognetti, A., 2016. Assessment of a smart sensing shoe for gait phase detection in level walking. Electronics, 5.4, 78.

Catalfamo, P., Moser, D., Ghoussayni, S., Ewins, D., 2008. Detection of gait events using an F-Scan in-shoe pressure measurement system. Gait \& posture, 28.3, 420-426.

Ciniglio, A., Guiotto, A., Spolaor, F., Sawacha, Z., 2021. The design and simulation of a 16-sensors plantar pressure insole layout for different applications: From sports to clinics, a pilot study. Sensors, 21.4, 1450.

Crea, S., Donati, M., De Rossi, S. M. M., Oddo, C. M., Vitiello, N., 2014. A wireless flexible sensorized insole for gait analysis. Sensors, 14.1, 1073-1093.

Della Croce, U., Cereatti, A., Mancini, M., 2018. Gait parameters estimated using inertial measurement units. In: Müller, B., Wolf, S. I., Brüggemann, G. P., Deng, Z., McIntosh, A. S., Miller, F., \& Selbie, W. S. (Eds), Handbook of human motion. Springer International Publishing, 245-265.

Hausdorff, J. M., Ladin, Z., Wei, J. Y., 1995. Footswitch system for measurement of the temporal parameters of gait. Journal of biomechanics, 28.3, 347-351.

Hopkins, D. W., 2001. What is a Norris derivative?. NIR news, 12.3, 3-5.

Kong, K., Tomizuka, M., 2009. A gait monitoring system based on air pressure sensors embedded in a shoe. In Proceedings of IEEE/ASME Transactions on mechatronics, 14.3, 358-370.

Mariani B, Rochat S, Büla CJ, Aminian K., 2012. Heel and toe clearance estimation for gait analysis using wireless inertial sensors. In Proceedings of the IEEE transactions on bio-medical engineering, 59.11, 3162-8.

Nove ${ }^{\circledR}{ }^{\text {Pedar }}{ }^{\circledR}$ System Web Page. Url: http://www.novel.de

Panebianco, G. P., Bisi, M. C., Stagni, R., \& Fantozzi, S., 2018. Analysis of the performance of 17 algorithms from a systematic review: Influence of sensor position, analysed variable and computational approach in gait timing estimation from IMU measurements. Gait \& posture, 66, 76-82.

Rochester, L., Mazzà, C., Mueller, A., Caulfield, B., McCarthy, M., Becker, C., ... \& Mobilise-D Consortium, 2020. A roadmap to inform development, validation and approval of digital mobility outcomes: the Mobilise-D approach. Digital Biomarkers, 4.1, 13-27.

Roth, N., Martindale, C. F., Eskofier, B. M., Gaßner, H., Kohl, Z., Klucken, J., 2018. Synchronized sensor insoles for clinical gait analysis in home-monitoring applications. Current Directions in Biomedical Engineering, 4.1, 433-437.

Skelly, M. M., Chizeck, H. J., 2001. Real-time gait event detection for paraplegic FES walking. In Proceedings of IEEE Transactions on neural systems and rehabilitation engineering, 9.1, 59-68. 
Smith, B. T., Coiro, D. J., Finson, R., Betz, R. R., McCarthy, J., 2002. Evaluation of force-sensing resistors for gait event detection to trigger electrical stimulation to improve walking in the child with cerebral palsy. In Proceedings of IEEE Transactions on Neural Systems and Rehabilitation Engineering, 10.1, 22-29.

Stork, M., 2003. Median filters theory and applications. In Proceedings of the third International Conference on Electrical and Electronics Engineering Papers-Chamber of Electrical Engineering, Bursa, Turkey, 1-5.

Tekscan ${ }^{\circledR}$ F-Scan ${ }^{\circledR}$ System Web Page. Url: http://www.tekscan.com

Trojaniello, D., Cereatti, A., Pelosin, E., Avanzino, L., Mirelman, A., Hausdorff, J. M., Della Croce, U., 2014. Estimation of step-by-step spatio-temporal parameters of normal and impaired gait using shank-mounted magneto-inertial sensors: application to elderly, hemiparetic, parkinsonian and choreic gait. Journal of neuroengineering and rehabilitation, 11.1, 1-12.

Whittle, Michael W., 1993. Gait analysis. In: Butterworth-Heinemann (Eds), The soft tissues., 187-199.

World Health Organization, 2007. International Classification of Functioning, Disability, and Health: Children \& Youth Version: ICF-CY. World Health Organization. 


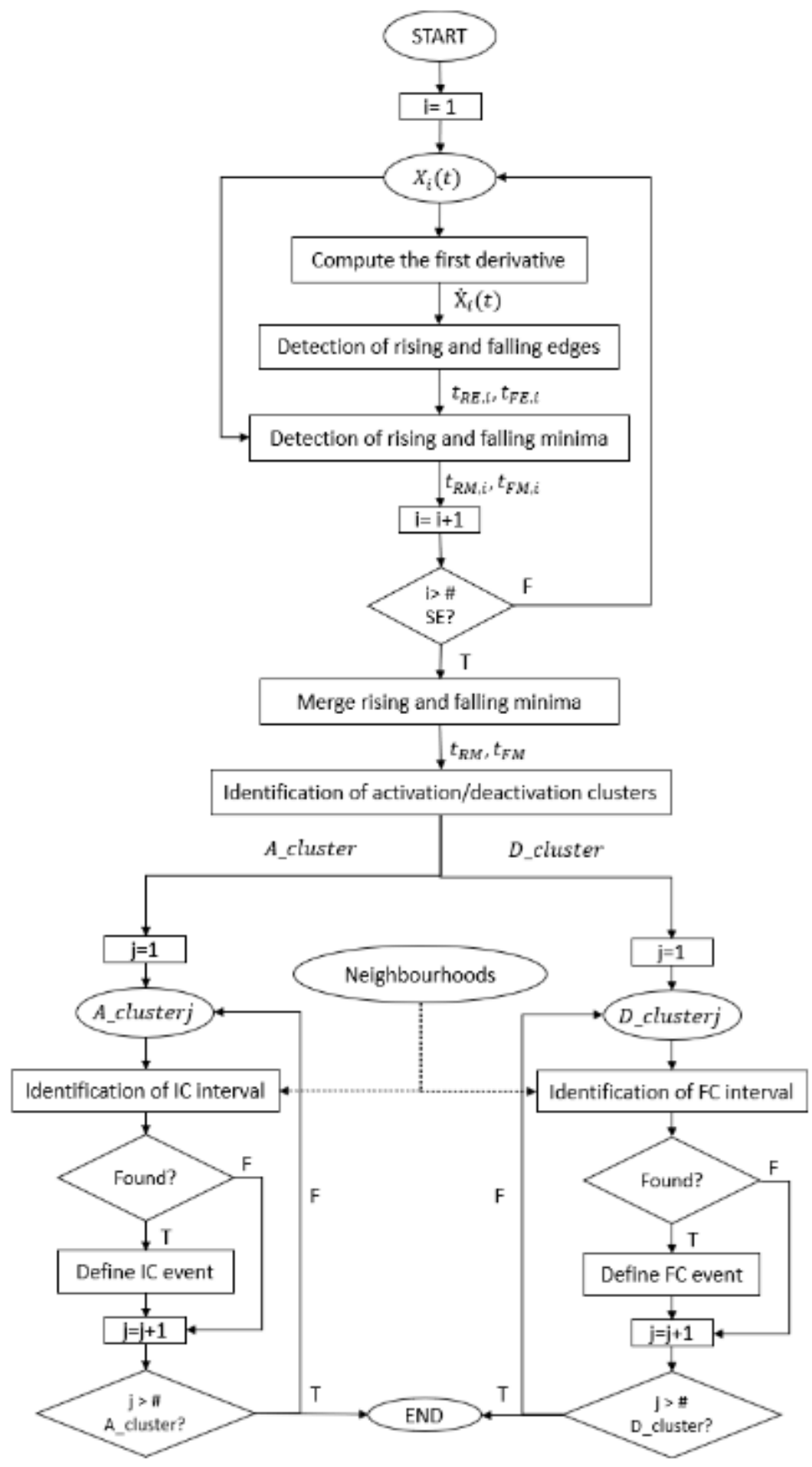

Figure A1. Algorithm workflow.

In Fig. A1 a detailed description of the algorithm workflow is illustrated.

Definitions:

$X_{i}(t)=$ pre-processed signal from the $\mathrm{i}$-th sensing element \#SE = number of sensing elements of the pressure insole

$\dot{\mathrm{X}}_{i}(t)=$ first derivative of $X p_{i}[n]$

$t_{R E, i}=$ rising edges instants

$t_{F E, i}=$ falling edges instants

$t_{R M, i}=$ rising minima instants 
$t_{F M, i}=$ falling minima instants

$t_{R E}=$ rising minima instants of all the sensing units

$t_{F E}=$ falling minima instants of all the sensing units

A_cluster $=$ activation clusters

D_cluster $=$ deactivation clusters

- Check on temporal distance. This is performed applying a threshold $T_{d}=0.6 \mathrm{~s}$. If the distance between consecutive events is lower than $T h_{d}$, the second event is discarded in case of rising edges, while the first event is discarded for the falling edges.

- Check on the amplitude reached by $x_{i}(t)$ after each rising edge instant and before each falling edge instant. The amplitude reached in the considered window (10 samples after a rising edge instant or 10 samples before a falling edge instant) must be at least $0.3 \mathrm{nu}$, otherwise the event is discarded. 
Appendix B

The neighbourhood of each sensing element is defined as reported in the following table:

\begin{tabular}{|c|c|}
\hline Sensing unit number & Neighbourhood \\
\hline 1 & $2,3,4,6,7$ \\
\hline 2 & $1,3,4,6,7$ \\
\hline 3 & $1,2,4,6,7,8,5$ \\
\hline 4 & $1,2,3,5,7,8,6,9$ \\
\hline 5 & $1,2,3,5,7,8,6,9$ \\
\hline 6 & $1,2,3,4,7,8$ \\
\hline 7 & $1,2,3,4,5,6,8,9$ \\
\hline 8 & $3,4,5,6,7,9,10$ \\
\hline 9 & $5,8,4,7,10,11$ \\
\hline 10 & $9,11,8,5,12$ \\
\hline 11 & $9,10,12,14,13,15,16$ \\
\hline 12 & $10,11,13,14,15,16$ \\
\hline 13 & $11,12,14,15,16$ \\
\hline 14 & $11,12,13,15,16$ \\
\hline 15 & $12,13,14,16,11$ \\
\hline 16 & $12,13,14,15,11$ \\
\hline & \\
\hline
\end{tabular}

